\title{
Immunity of honeybee guards reflects their transition from house bees to foragers
}

Federico CAPPA1,*, IACOPO Petrocelli1, Alessandro Cini1,2, Irene PePiciello1, Michele Giovannini1, AnNa Marta LazZeri1, BRUnella Perito1, Stefano TurillaZZI1 and Rita CERVO1

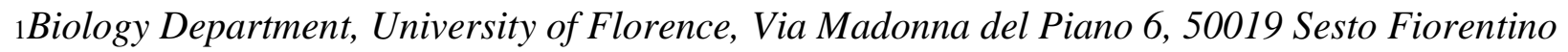
(Florence), Italy

${ }_{2}$ Centre for Biodiversity and Environment Research, University College London, Gower Street, London WCIE 6BT, UK

*Corresponding author: Federico Cappa, Dipartimento di Biologia, Università degli Studi di Firenze, Via Madonna del Piano 6, 50019 Sesto Fiorentino (Firenze), Italy (E-mail: federico.cappa@unifi.it).

Eusocial insect colonies represent some of the most extreme examples of specialized division of labor. Ageing in workers is often associated with a temporal polyethism in the tasks performed both inside and outside the colony. Such behavioral transition is sometimes linked to a gradual reduction in individual immunity. Here, we studied the immune ability of Apis mellifera guard bees, which represent an intermediate stage between house bees working inside the nest and foragers collecting resources outside, to assess if their specific task is associated with an immune specialization. Through immune challenge with Gram-negative bacteria Escherichia coli, we compared the guards ability to clear bacterial cells from their haemolymph with respect to house bees and foragers. Our findings demonstrate that guards do not show an immune specialization linked to their task but seem to represent a transition also in terms of immunity, since their anti-bacterial response appears intermediate between house bees and foragers.

KEY WORDS: Apis mellifera, division of labor, bacterial clearance, Escherichia coli, immune challenge.

Division of labor, i.e. the pattern of specialization by cooperative individuals performing different tasks or roles in a social group, is often associated with physiological and/or morphological correlates 
that improve task performance (Duarte et al. 2011). Honeybee workers have represented for decades a model to study social and physiological implications of division of labor in social Hymenoptera (Robinson 1992) with a growing attention towards the linkage between temporal polyethism and immunity (Amdam et al. 2005; Wilson-Rich et al. 2008; Laughton et al. 2011). Among the different task specialization of honeybee workers, guards represent a peculiar behavioral phenotype, being intermediate between house bees working inside the nest and foragers collecting resources outside (Moore et al. 1987). Workers become guards after performing in-hive duties but before foraging: they patrol the colony entrance, inspecting incoming bees and excluding foreign individuals (Breed et al. 2004; Cappa et al. 2014, 2016, 2019). Only a small proportion of the colony worker population (about 10\%) performs guarding activities (Breed et al. 2004), usually middle-aged workers, between 7 and 22 days post-emergence (Moore et al. 1987).

45 Given their physiological and behavioral features, guards represent an interesting model to investigate the association between immune system and specific task performed. Indeed, from a physiological point of view, juvenile hormone $(\mathrm{JH})$ titers of guards are reported to be higher than other middle-aged bees and JH seems to be involved in the regulation of aggressiveness (Huang et al. 1994; Pearce et al. 2001). The high level of circulating JH in guards' haemolymph may have immunosuppressive effects as in foragers (Amdam et al. 2004, 2005), resulting in a weaker immune system due to these physiological correlates. Thus, guards could be less immunocompetent than house bees and more similar to foragers in their immunity. From an ultimate perspective, attending to the colony queen and brood is a delicate task and an enhanced immunity in house bees with respect to guards and foragers working outside the colony social core could represent an evolutionary adaptation at the colony level to reduce the risk of pathogen transmission to the queen and the vulnerable brood (Cremer et al. 2007). However, foraging outside the colony could expose foragers to pathogens (Durrer \& Schimd-Hempel 1994), and interacting with incoming foragers at the hive entrance might also represent a costly task in terms of immunity since frequent interactions with conspecifics increase the risk of disease transmission (Cremer et al. 2007, 2018). Under this perspective we may expect 
guards and foragers to show a strong immune response to cope with the potential higher risks linked to their specific task. In this scenario, the present work aims to compare the immune ability of guards with that of house bees and foragers in order to understand if their specialized task is associated with their individual immunity.

\section{MATERIALS AND METHODS}

\section{Insect collection and maintenance}

Apis mellifera ligustica workers were collected from three hives housed outside the Department of Biology at the University of Florence, Sesto Fiorentino (Florence, Central Italy).

To obtain house bees of known age, combs with sealed brood were removed from hives and transferred to the laboratory. Newly-emerged workers were marked with a spot on the thorax with different paint markers according to day of collection and hive of origin before being reinserted into their hives. Around 300 newly-emerged workers per hive were marked every week for 4 weeks. Before the immune challenge, combs were inspected to collect marked workers of an age span between 1 and 2 weeks post-emergence. With this procedure we obtained house bees of an age interval similar to guards (Moore et al. 1987). Since guards do not go back to inside-hive duties after initiating guarding (Breed et al. 2004), we assumed that house bees collected on the combs were not guards at the time of collection. Marking was used to identify house bees among workers inside the crowded hive. Foragers and guards were instead collected while performing their specific tasks. Unfortunately it was not possible to control for the exact age of guards because, as reported by previous work, the percentage of workers that become guards is quite low (about 10\%) and in fact, only a couple of our marked bees were observed while involved in guarding tasks (and collected for the bacterial injection). Similarly, the possibility to collect a sufficient number of marked foragers after 3 weeks was quite scarce, but workers performing foraging tasks should be at least 18-20 days old (Seeley 1982). Thus, we collected at the entrance of each hive a large number of unmarked foragers returning from their foraging. Finally, bees at the entrance were observed for 20 min every 
day prior to immune challenge and classified as guards if they showed typical guarding behavior at the hive entrance: patrolling the board with wings held open, chasing landing bees, inspecting bees on the board and attacking some of them (Butler \& Free 1952). All the bees performing such behaviors were collected and transferred to the lab for the immune challenge.

\section{Bacterial injection}

To evaluate the workers' ability to remove bacteria from their haemolymph (i.e. bacterial clearance), we challenged workers with the Gram-negative bacteria Escherichia coli, an immune elicitor not naturally found in A. mellifera, to exclude its presence prior to experimental infection, already used in previous studies testing immunocompetence in insects (Yang \& Cox-Foster 2005; Manfredini et al. 2010; Cappa et al. 2015; 2019). Injection of live bacteria induce the activation of the organism immune system (Charles \& Killian 2015) and subsequent bacterial clearance evaluation provide an integrative view of workers immunity being linked to other parameters used to assess insects' immune response (Gillespie et al. 1997). Bacterial cultures of E. coli tetracycline-resistant strain XL1-Blue were grown in Luria-Bertani (LB) complex medium added with $10 \mu \mathrm{g} / \mathrm{mL}$ tetracycline overnight at $37^{\circ} \mathrm{C}$ in a shaking incubator. After centrifugation, bacteria were washed twice and then resuspended in phosphate-buffered saline (PBS). The approximate amount of bacterial cells in the solution was determined using a hemocytometer (Neubauer), then cells were diluted to $\sim 1.5 \times 108$ cells $/ \mathrm{mL}$ in PBS. Bees were injected with $1 \mu \mathrm{L}$ of inoculum, containing $\sim 1.5 \times 10$ s cells, between the 2 nd and 3rd tergite with a HamiltonTm micro-syringe (Cini et al. 2018). After injection, bees were separated according to category into plastic cylindrical containers $(\varnothing 10 \mathrm{~cm} \times \mathrm{h} 10 \mathrm{~cm})$ provided with ad libitum honey as food and maintained under controlled conditions $\left(\sim 30{ }^{\circ} \mathrm{C} ; 55 \% \mathrm{RH}\right)$. Twenty-four hours later workers were dissected in a plate on ice to facilitate manipulation and removal of the sting apparatus in order to avoid a reduction in bacteria viable cells due to antimicrobial activity of venom compounds (Baracchi et al. 2011). Each dissected bee was then inserted into a sterile plastic bag with 
the bee body in the PBS. Afterwards, $0.1 \mathrm{~mL}$ of serially diluted PBS suspensions (dilutions $10_{-1}, 10$ -

2) of each sample were plated on LB solid medium added with $10 \mu \mathrm{g} / \mathrm{mL}$ tetracycline and incubated overnight at $37^{\circ} \mathrm{C}$. The following day, colonies grown on the plate were counted and expressed as

Colony Forming Units (CFUs) per bee. A total of 348 E. coli-injected bees were plated: (i) house bees, $N=176$, (ii) guards, $N=85$, (iii) foragers, $N=87$. At least 15 workers of each category per colony were used. Ten control bees for each category $(N=30)$ were injected with $1 \mu \mathrm{L}$ of PBS, homogenized and plated following the same procedure to ensure absence of bacterial strains capable of growing on LB plates added with tetracycline.

\section{Statistical analyses}

To test the effects of bee category on the antibacterial response we fitted a generalized linear model (GLZ) with negative binomial distribution with log-link function and using Type III Sums of squares for accounting for unbalanced design. Hive of origin and interaction between bee category and hive were included as model effects. Sequential Bonferroni-corrected pairwise comparisons were performed to test for difference among bee categories $(\alpha=0.017)$. Statistical analyses were performed using the program SPSS 17.0 for Windows (SPSS Inc., Chicago, IL, USA).

\section{RESULTS}

Antibacterial response was significantly different according to bee category (GLZ: Wald $\chi^{2}=7.303$, $\mathrm{df}=2, P=0.026$ ) while neither hive of origin nor the interaction between hive of origin and bee category were significant (respectively GLZ: Wald $\chi^{2}=0.307, \mathrm{df}=4, P=0.858$ and GLZ: Wald $\chi^{2}=$ 5.567, $\mathrm{df}=2, P=0.234$ ). Bacterial clearance was higher in house bees than in foragers (Fig. 1, Wald $\chi^{2}=7.153, \mathrm{df}=2, P=0.015, \alpha=0.017$, effect size: Cohen's $\left.\mathrm{d}=0.202\right)$, while no significant difference was found between guards and house bees (Fig. 1 , Wald $\chi^{2}=0.248, \mathrm{df}=2, P=0.618, \alpha=0.017$ ) and between guards and foragers (Fig. 1 , Wald $\chi^{2}=3.556, \mathrm{df}=2, P=0.119, \alpha=0.017$ ). No CFUs were detected in plates from PBS-injected controls. 


\section{DISCUSSION}

140 Our results indicate that the behavioral task transition of guards from non-guarding house bees to

141 foragers is paralleled by a transition in individual immunity, with guards having an intermediate 142 antibacterial response between workers performing tasks inside the hive and foragers collecting 143 resources outside the colony. Guards did not show an increased immune function despite their 144 behavioral specialization, their antimicrobial response being similar when compared to both house 145 bees and foragers. From a proximate point of view, we can hypothesize that the physiological 146 correlates of guarding, i.e. high JH and lowered vitellogenin titers (Huang et al. 1994; Pearce et al. 147 2001) together with the energetically demanding task of a continuous patrolling at the colony entrance 148 could be responsible for the absence of an enhanced immunity in guards. On the other hand, from an 149 ultimate perspective, guarding may not represent a costly task in terms of social immunity and would 150 not require therefore an enhanced immune system in workers performing such behavior. A high 151 number of interactions with incoming foragers at the hive entrance might expose guards to a higher 152 risk of disease transmission (Cremer et al. 2007). However, guards in the majority of cases guard 153 briefly, for 1 to 3 days, and they do not revert to inside-hive duties after initiating guarding activities 154 (Moore et al. 1987; Breed et al. 2004). Thus, guarding for a short period of time may not require a task-related increase in individual immune ability.

The immune trend showed by our groups of bees highlighted a higher immune ability in bees working inside the nest as already demonstrated by previous work (Amdam et al. 2004, 2005; Laughton et al. 158 2011). Workers in the first 2 weeks after eclosion are usually engaged in queen attendance and nursing 159 activities such as brood care and brood rearing (Huang et al. 1994). Nurses are also the main target 160 of Varroa mites (Cervo et al. 2014; Xie et al. 2016), and their increased immunity could help them 161 to cope with the parasite pressure and the pathogens it transmits (Francis et al. 2013).

162 The intermediate immune response of guards and the progressive trend of immunity loss going from 163 inside-nest to outside-nest tasks suggest that, as postulated by social immunity predictions (Cremer 
et al. 2007, 2018), individuals working in the social core of the colony have highly efficient immune responses while immune competence decrease while approaching energetically costly outside-nest duties.

\section{ACKNOWLEDGMENTS}

169 The authors thank Dr M. Calosi for assistance in the laboratory. The authors also thank two anonymous reviewers for their helpful comments on the manuscript.

\section{DISCLOSURE STATEMENT}

173 No potential conflict of interest was reported by the authors.

FUNDING

176 Financial support was provided by project PRIN2012 (prot. 2012RCEZWH_001). welfare laws.

\section{AUTHOR CONTRIBUTION}

F. Cappa and R. Cervo conceived and designed the research. F. Cappa, A. Cini, I. Petrocelli, I. 184 Pepiciello, M. Giovannini and A.M. Lazzeri performed the assays and collected the data. A. Cini 185 analysed the data. F. Cappa wrote the manuscript. R. Cervo, S. Turillazzi and B. Perito provided facilities and materials. All authors read and approved the manuscript.

\section{REFERENCES}


Amdam GV, Aase ALT, Seehuus SC, Fondrk MK, Norberg K, Hartfelder K. 2005. Social reversal of immunosenescence in honey bee workers. Exp Gerontol. 40:939-947.

Amdam GV, Simões ZL, Hagen A, Norberg K, Schrøder K, Mikkelsen $\varnothing$, Kirkwood TB, Omholt SW. 2004. Hormonal control of the yolk precursor vitellogenin regulates immune function and longevity in honeybees. Exp Gerontol. 39:767-773.

Baracchi D, Francese S, Turillazzi S. 2011. Beyond the antipredatory defence: honey bee venom function as a component of social immunity. Toxicon. 58(6-7):550-557.

Breed MD, Guzmán-Novoa E, Hunt, GJ. 2004. Defensive behavior of honey bees: organization, genetics, and comparisons with other bees. Annu Rev Entomol. 49:271-298.

Butler CG, Free JB. 1952. The behaviour of worker honeybees at the hive entrance. Behaviour. 4: 262-291.

Cappa F, Beani L, Cervo R, Grozinger C, Manfredini F. 2015. Testing male immunocompetence in two hymenopterans with different levels of social organization:'live hard, die young?'. Biol J Linn Soc. 114:274-278.

Cappa F, Bruschini C, Protti I, Turillazzi S, Cervo R. 2016. Bee guards detect foreign foragers with cuticular chemical profiles altered by phoretic varroa mites. J Api Res. 58:268-277.

Cappa F, Petrocelli I, Dani FR, Dapporto L, Giovannini M, Silva-Castellari J, Turillazzi S, Cervo R. 2019. Natural biocide disrupts nestmate recognition in honeybees. Sci Rep. 9:3171. Assessing immunocompetence in red palm weevil adult and immature stages in response to bacterial 
222 Cervo R, Bruschini C, Cappa F, Meconcelli S, Pieraccini G, Pradella D, Turillazzi S. 2014. High

223 Varroa mite abundance influences chemical profiles of worker bees and mite-host preferences. J Exp

224 Biol. 217:2998-3001

225

226 Charles HM, Killian KA. 2015. Response of the insect immune system to three different immune 227 challenges. J Insect Physiol. 81:97-108.

228

229 Cini A, Cappa F, Petrocelli I, Pepiciello I, Bortolotti L, Cervo R. 2018. Competition between the 230 native and the introduced hornets Vespa crabro and Vespa velutina: a comparison of potentially 231 relevant life - history traits. Ecol Entomol. 43:351-362.

Cremer S, Armitage SAO, Schmid-Hempel P. 2007. Social immunity. Curr Biol. 17:R693-R702.

Cremer S, Pull CD, Fürst, MA. 2018. Social immunity: Emergence and evolution of colony-level disease protection. Annu Rev Entomol. 63:105-123.

Duarte A, Weissing FJ, Pen I, Keller L. 2011. An evolutionary perspective on self-organized division

Durrer S, Schmid-Hempel P. 1994. Shared use of flowers leads to horizontal pathogen transmission.

Francis RM, Nielsen SL, Kryger P. 2013. Varroa-virus interaction in collapsing honey bee colonies.

Gillespie JP, Kanost MR, Trenczek T. 1997. Biological mediators of insect immunity. Annu Rev Entomol. 42:611-643.

Huang ZY, Robinson GE, Borst DW. 1994. Physiological correlates of division of labor among 
256 Manfredini F, Beani L, Taormina M, Vannini L. 2010. Parasitic infection protects wasp larvae against 257 a bacterial challenge. Microbes Infect. 12:727-735.

Moore AJ, Breed MD, Moor MJ. 1987. The guard honey bee; ontogeny and behavioural variability of workers performing a specialized task. Anim Behav. 35:1159-1167.

Pearce AN, Huang ZY, Breed MD. 2001. Juvenile hormone and aggression in honey bees. J Insect

Robinson GE. 1992. Regulation of division of labor in insect societies. Annu Rev Entomol. 37:637266 665.

Seeley TD. 1982. Adaptive significance of the age polyethism schedule in honeybee colonies. Behav 270

Xie X, Huang ZY, Zeng Z. 2016. Why do Varroa mites prefer nurse bees? Sci Rep. 6:28228. doi: $10.1038 /$ srep 28228

Yang X, Cox-Foster DL. 2005. Impact of an ectoparasite on the immunity and pathology of an invertebrate: evidence for host immunosuppression and viral amplification. Proc Nat Acad Sci USA. 102:7470-7475.

Wilson-Rich N, Dres ST, Starks PT. 2008. The ontogeny of immunity: development of innate immune strength in the honey bee (Apis mellifera). J Insect Physiol. 54:1392-1399.

Fig. 1 - Bacterial clearance of $E$. coli-injected workers. House bees (HB, $N=176)$ showed a significantly higher antibacterial response than foragers $(\mathrm{F}, N=87)$, while guards $(\mathrm{G}, N=85)$ showed a bacterial clearance similar to both house bees and foragers. Box plots represent the number of colony forming units (CFUs) detected on LB agar plates from the homogenized honeybee suspension 286 